\title{
Continuous tuning of the threshold voltage of organic thin-film transistors by a chemically reactive interfacial layer
}

\author{
Harald Etschmaier • Peter Pacher • Alexandra Lex • \\ Gregor Trimmel • Christian Slugove · Egbert Zojer
}

Received: 24 September 2008 / Accepted: 24 November 2008 / Published online: 23 December 2008

(C) The Author(s) 2008. This article is published with open access at Springerlink.com

\begin{abstract}
For the design and manufacture of complex integrated circuits, control over the threshold voltage of the transistors is essential. In the present contribution, we present a non-invasive method to tune the threshold voltage of organic thin-film transistors after device assembly over a wide range without any significant degradation of the device characteristics. This is realized by incorporating a thin, chemically reactive siloxane layer bonded to the gate oxide. This results in threshold voltages of around $70 \mathrm{~V}$ in the as-prepared devices. By exposing a transistor modified in this way to ammonia at different concentrations, the threshold voltage can be tuned in steps of only a few volts. This treatment affects only the charge density at the semiconductor-dielectric interface, leaving the overall shape of the transistor characteristics and the charge-carrier mobility largely unaltered.
\end{abstract}

PACS 81.05.Hd · 85.30.De · 85.30.Tv · 81.16.Dn •

73.40.Qv $\cdot$ 73.61.Ph $\cdot$ 07.07.Df

H. Etschmaier · P. Pacher $(\bowtie) \cdot$ E. Zojer

Institute of Solid State Physics, Graz University of Technology,

Petersgasse 16, 8010 Graz, Austria

e-mail: peter.pacher@tugraz.at

E. Zojer $(\bowtie)$

e-mail: egbert.zojer@tugraz.at

A. Lex · G. Trimmel $\cdot$ C. Slugovc

Institute for Chemistry and Technology of Materials, Graz

University of Technology, Stremayrgasse 16, 8010 Graz, Austria

Present address:

A. Lex

Institute of Physical Chemistry, University of Münster,

Correnstrasse 28/30, 48149 Münster, Germany

\section{Introduction}

Organic thin-film transistors (OTFTs) are considered to be of great importance for flexible, large-area, and low-cost electronic devices [1]. A critical property of integrated circuits is the power dissipation, because heating the device limits operating speed, potential for miniaturization, and also battery life. In digital circuits, power consumption is proportional to the square of the supply voltage [2]. However, reducing the supply voltage to less than three times the threshold voltage results in a remarkable slow down of circuit operation, due to a reduction of the source-drain current [3]. Therefore, the threshold voltage must be reduced along with the supply voltage. The lower limit threshold voltage is set by the amount of off-state leakage current. A threshold voltage, which is too small, will cause high leakage currents in the off-state at zero gate volt. Thus, a good control of the threshold voltage is essential if the power dissipation is to be minimized. For complementary logic circuits, which have many advantages in terms of high noise immunity and low static power consumption, threshold voltage control is especially important. The threshold voltage difference between $\mathrm{p}$ - and n-type transistors causes operating margin suppression and performance degradation [4]. Tuning of at least one of the transistor types is necessary to obtain a balanced circuit, with the same threshold voltage for both transistor types. In silicon metal-oxidesemiconductor field-effect transistors, which are used in mainstream electronic devices, the threshold voltage can be influenced by adjusting the doping level of the semiconductor [5].

For organic electronics, different approaches have to be explored. In this context, dual-gate architectures have been used to realize variable threshold voltages $[6,7]$ and transistors with liquid contacts and, therefore, tunable character- 
istics have been presented [8]. Another possibility is to influence the density of charged interface states on the dielectric. This has been achieved by modifying a parylene gate dielectric with oxygen plasma [9] or a UV-ozone [10] treatment, or by inserting a layer of metal oxide in the transistor structure [11]. Also, thin siloxane layers have been found to significantly modify the threshold voltage $[12,13]$.

\section{Controlling the threshold voltage with reactive thin layers}

In this contribution, we present a non-invasive method to tune the threshold voltage after the assembly of the device over a wide range without any significant degradation of the transistor characteristics. It is realized by incorporating a thin, chemically reactive siloxane layer bonded to the gate oxide. It consists of commercially available molecules bearing sulfonic acid (30\%) and sulfonyl chloride (70\%) groups. The sulfonic acid substituents protonate the organic semiconductor at the interface. This leads to a high charge-carrier concentration in the conducting channel in close proximity to the dielectric (see Fig. 1) [14, 15]. As most of the charge transport in the channel of an organic TFT takes place in this region [16, 17], additional charges at that interface strongly affect the characteristics of the transistor. Consequently, the device is switched on at zero gate bias and a relatively high positive voltage of around $+70 \mathrm{~V}$ is necessary to deplete the channel. In this case, the device is operating in depletion mode.

The interfacial layer used has been shown to chemically react with amines [18]. The acidic groups at the semiconductor-dielectric interface are neutralized by the alkaline ammonia, resulting in the formation of the corresponding ammonium sulfonate (the sulfonic acid chloride groups react with ammonia to the corresponding sulfonamides [18], which, however, play no role for the device characteristics as shown in Ref. [14]). Excess amounts of ammonia thus cancel the doping effect by suppressing the ability of the sulfonic acid functionalities to protonate the overlying semiconductor. Consequently, after exposure of the device to ammonia, the voltage necessary to deplete the channel is lowered and can even go to negative voltages, making the device operate in accumulation mode. In the present contribution, we make use of the above-described interface chemistry to realize devices in which the threshold voltage can be set to an arbitrary value between +70 and $-20 \mathrm{~V}$ by controlled exposure to ammonia.

\section{Experimental realization}

As substrates for the transistors, $\mathrm{n}$-type doped silicon wafers with a thermally grown 165 -nm-thick silicon oxide layer were used. The doped silicon acts as a gate electrode and the silicon oxide as the dielectric. The wafers were provided by Siegert Consulting in an industrially precut size of $2 \times 2 \mathrm{~cm}^{2}$. In a first step, they were cleaned by radio frequency oxygen plasma etching at a pressure of $0.5 \mathrm{mbar}$ for $35 \mathrm{~s}$. Thereafter, they were further cleaned in an ultrasonic bath with ultra-pure water and dried with a stream of $\mathrm{CO}_{2}$ gas. To avoid any contamination with water, the wafers were additionally stored under vacuum for about $15 \mathrm{~min}$. This pretreatment causes a hydrophilic surface with a high concentration of hydroxyl groups [18] needed as attachment points for the siloxane-based reactive layer.

For the assembly of the reactive layer, the samples were immersed in a 0.1 vol. $\%$ solution of 4 -(2-(trichlorosilyl)ethyl)benzene sulfonic acid chloride (T-SC) containing
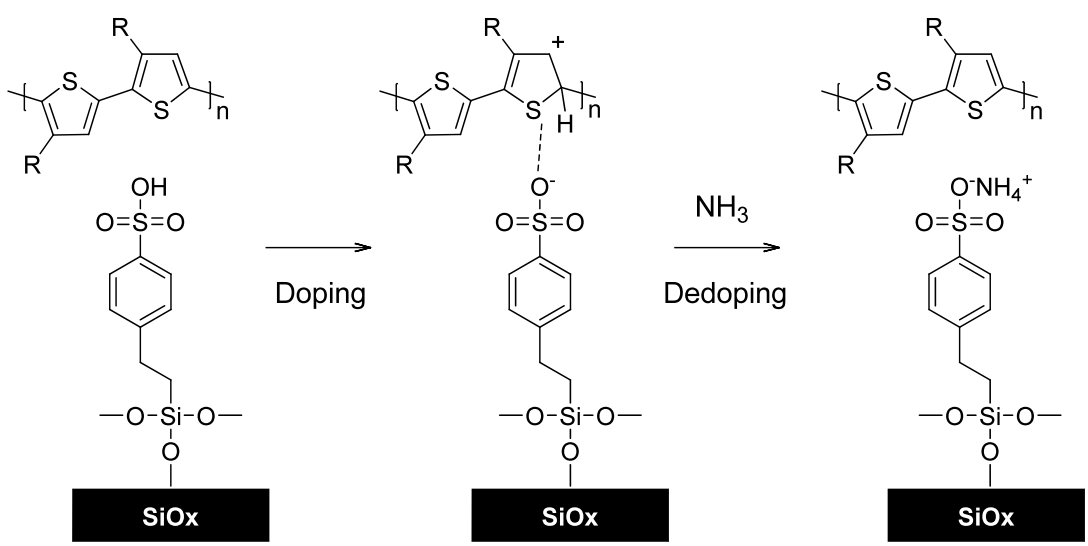

Fig. 1 Chemical doping and dedoping mechanism suggested for a sulfonic acid functionality at the organic semiconductor/dielectric interface according to Ref. [14]. The acidic groups attached to the siloxanes are protonating the overlying rr-P3HT, making the channel conducting at moderately positive gate voltages. Upon contact with ammonia, the acidic groups are neutralized and the effect is suppressed, shifting the threshold voltages to lower values 
$30 \%$ of the free sulfonic acid analogue (T-SA) in anhydrous toluene. The chemicals were purchased from ABCR. To allow a homogeneous layer formation, the wafers were kept in this solution for about $16 \mathrm{~h}$. The whole process was done under inert-gas conditions in an argon glove box. In this way, the solution-phase oligomerization of the trichlorosilane groups by an excessive amount of water resulting in increased film thicknesses was largely avoided [18]. Thereafter, the samples were cleaned for $2 \mathrm{~min}$ in an ultrasonic bath in pure toluene and then rinsed with toluene and dried with a stream of $\mathrm{CO}_{2}$ gas. Subsequently, they were annealed at $100^{\circ} \mathrm{C}$ and $10^{-2}$ mbar for $30 \mathrm{~min}$. This procedure typically results in interfacial layers with thicknesses in the range from 2.0 to $2.6 \mathrm{~nm}$ [18] with the actual thickness being influenced by minute amounts of residual water in the production process.

After annealing, the wafers were transferred back into the argon glove box and a solution of regio-regular poly(3hexylthiophene) (rr-P3HT) in water-free spectroscopy grade chloroform $(2 \mathrm{~g} / \mathrm{L})$ was spin cast. The polymer was purchased as Plexcore OS 1100 from Sigma Aldrich and has a molecular weight of $25000 \mathrm{u}$ and a polydispersity of about 2 . $100 \mu \mathrm{L}$ of the solution were spread on the surface and after that the spin coater was immediately switched on for $40 \mathrm{~s}$ at a speed of $1600 \mathrm{rpm}$. Then, the samples were dried for $5 \mathrm{~min}$ at $50^{\circ} \mathrm{C}$. Subsequently, 20 -nm-thick gold contacts were evaporated through a shadow mask (channel length of $25 \mu \mathrm{m}$ and channel width of $7 \mathrm{~mm}$ ) at a base pressure below $10^{-6}$ mbar. A schematic picture of the resulting transistor structure and dimensions is shown in Fig. 2.

To expose the device to ammonia, the transistor was mounted in a measuring cell attached to a gas-mixing system. The argon (5.0 purity) and the ammonia (4.5 purity) used in this setup were provided by Linde Gas. Two rotary flow meters (Krohne DK 800) with integrated needle valves allow adjusting the flow of both gases separately. After the flow meters, the pipes are joined together to allow mixing of

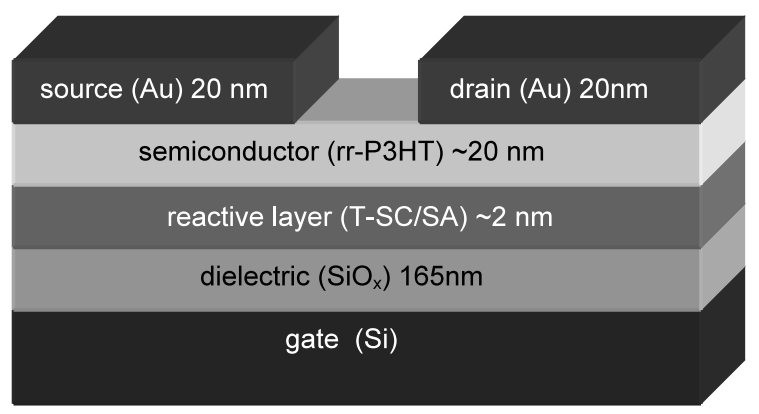

Fig. 2 Schematic structure of the bottom-gate, top-contact devices used. A heavily doped silicon wafer is used as substrate and also as gate contact. $165 \mathrm{~nm}$ of silicon oxide are thermally grown on top to serve as a dielectric. A chemically reactive thin layer of T-SC/SA is deposited from solution; the semiconducting rr-P3HT is spin cast and the gold contacts are evaporated through a shadow mask the gases. To minimize the dead volume of the pipes, valves to close the gas flow in each of the branches are located in the immediate vicinity of that joint. The argon flow meter allows changing the flow between 10 and $100 \mathrm{~L} / \mathrm{h}$ and the ammonia flow meter similarly between 1 and $10 \mathrm{~L} / \mathrm{h}$. The transistors are contacted inside the cell and connected to a parameter analyzer (Agilent Technologies E5262A) for electrical characterization. Each measurement consists of output characteristics at seven different gate voltages between $-60 \mathrm{~V}$ and $+60 \mathrm{~V}$ and transfer characteristics at drain voltages between $-2 \mathrm{~V}$ and $-45 \mathrm{~V}$. All characteristics reported here have been recorded after exposure to ammonia and subsequent to extensive flushing of the cell with argon for at least $10 \mathrm{~min}$.

\section{Results and discussion}

Figure 3 shows the transfer characteristics of a device with the above-described structure brought into contact with various concentrations of ammonia in argon at a drain voltage of $-45 \mathrm{~V}$. The ammonia concentrations used were: $\approx 1$ vol. $\%$ (1:100), $\approx 2$ vol.\% (2:100), 4.8 vol.\% (5:100), 9.1 vol.\% (10:100), 16.7 vol.\% (10:50), and pure ammonia (100\%)

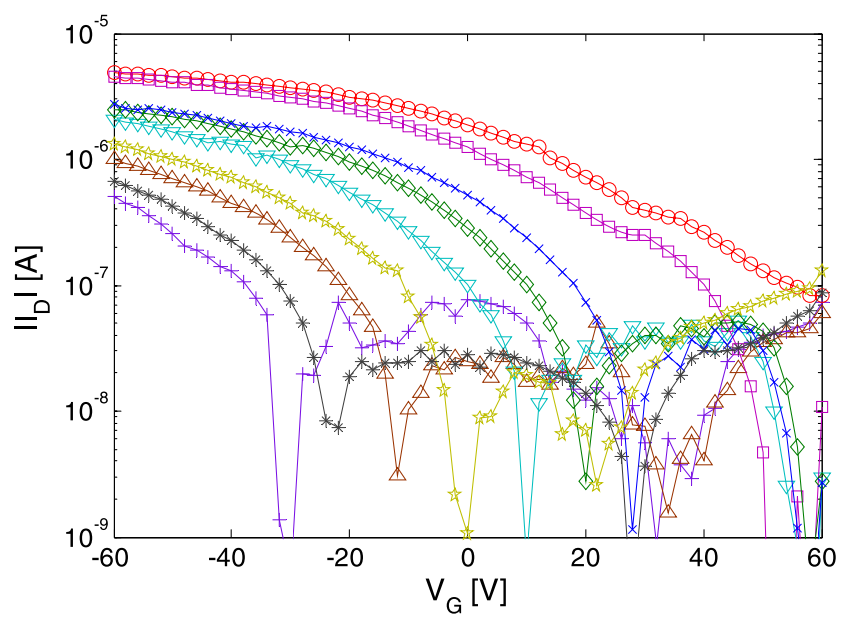

Fig. 3 Transfer characteristics of a rr-P3HT OTFT containing a reactive T-SC/SA layer after successive exposures to ammonia at different concentrations at a source-drain voltage of $-45 \mathrm{~V}$. The channel of the pristine device is heavily doped and can hardly be depleted. Upon contact with ammonia, the doping is (partially) compensated and the characteristics are shifted towards lower gate voltages (red circles: prior to exposure to $\mathrm{NH}_{3}$, purple rectangles: after exposure to ammonia to argon ratio of 1:100 (a flow of $1 \mathrm{~L} / \mathrm{h} \mathrm{NH} \mathrm{NH}_{3}$ and $100 \mathrm{~L} / \mathrm{h} \mathrm{Ar}$ ) for $1 \mathrm{~min}$, blue x's: after $1 \mathrm{~min}$ of a 2:100 mixture, green diamonds: after $1 \mathrm{~min}$ of a 5:100 mixture, turquoise triangles: after $1 \mathrm{~min}$ of a 10:100 mixture, yellow stars: after 1 min of a 10:50 mixture, brown triangles: after 1 min of a 10:0 mixture, black crosses: after $10 \mathrm{~min}$ of a 10:0 mixture, violet pluses: after another $10 \mathrm{~min}$ of a 10:0 mixture). All characteristics shown in this figure have been obtained after extensively flushing the cell with argon for at least $10 \mathrm{~min}$ and are reported for the same device exposed in successive steps 
Table 1 Threshold voltages, $V_{\mathrm{T}}$, charge-carrier mobilities, $\mu_{\mathrm{sat}}$, and charge densities at the interface, $\sigma_{\mathrm{if}}$, for rr-P3HT-based OTFTs containing a T-SC/SA interfacial layer after exposure to various doses of ammonia. The charge-carrier mobilities, as well as the threshold voltages, were extracted in the saturation region. The interface charge den- sity was calculated for each value of the threshold voltage assuming a specific capacity of the dielectric of $209 \mu \mathrm{F} / \mathrm{m}^{2}$. All values shown in this table have been obtained after extensively flushing the cell with argon for at least $10 \mathrm{~min}$ and are reported for the same device exposed in successive steps

\begin{tabular}{|c|c|c|c|c|}
\hline $\mathrm{NH}_{3}:$ Ar flow $(\mathrm{L} / \mathrm{h})$ & $\begin{array}{l}\mathrm{NH}_{3} \text { to } \mathrm{Ar} \\
\text { concentration }\end{array}$ & $V_{\mathrm{T}}(\mathrm{V})$ & $\mu_{\text {sat }}\left(10^{-4} \mathrm{~cm}^{2} / \mathrm{V} \mathrm{s}\right)$ & $\sigma_{\text {if }}\left(10^{-3} \mathrm{C} / \mathrm{m}^{2}\right)$ \\
\hline Pristine & 0 vol. $\%$ & 71 & 1.2 & 14.7 \\
\hline $1 \mathrm{~min} \quad 1: 100$ & 1 vol. $\%$ & 55 & 1.4 & 11.5 \\
\hline$+1 \min 2: 100$ & 2 vol. $\%$ & 38 & 1.1 & 7.9 \\
\hline$+1 \min 5: 100$ & 4.8 vol. $\%$ & 28 & 1.3 & 5.8 \\
\hline$+1 \min 10: 100$ & 9.1 vol. $\%$ & 17 & 1.3 & 3.5 \\
\hline$+1 \min 10: 50$ & 16.7 vol. $\%$ & 5 & 1.1 & 1.1 \\
\hline$+1 \min \quad 10: 0$ & 100 vol. $\%$ & 0 & 1.0 & 0.0 \\
\hline$+10 \min 10: 0$ & 100 vol. $\%$ & -16 & 1.2 & \\
\hline$+10 \min 10: 0$ & 100 vol. $\%$ & -21 & 1.1 & \\
\hline
\end{tabular}

(10:0). They were realized by the argon and ammonia flows (in $\mathrm{L} / \mathrm{h}$ ) given in the parentheses. Each device was successively exposed to the above fluxes for $1 \mathrm{~min}$ each and finally twice to pure $\mathrm{NH}_{3}$ for 10 min (compare also Table 1). The pristine device is switched on over the whole measured gate voltage region (i.e. the turn-on voltage is clearly above $60 \mathrm{~V}$ ). After each ammonia exposure, the transfer characteristics of the device is shifted towards lower gate voltages. The overall shape of the characteristics remains, however, virtually unchanged. In particular, no deterioration (i.e. increase) of the off current is observed. The turn-on voltage, which can be estimated directly from this plot, decreases by several volts upon each step as a result of the exposure to higher ammonia concentrations.

The output characteristics of the device after different ammonia exposures at gate voltages of $-40 \mathrm{~V}$ and $0 \mathrm{~V}$ are reported in Fig. 4. At a gate voltage of $-40 \mathrm{~V}$, the device is switched on for all stages of the reaction at the interface, while at $0 \mathrm{~V}$ gate voltage the device is switched off ( $I_{\mathrm{D}} \ll 1 \times 10^{-7}$ A over the whole $V_{\mathrm{D}}$ range) after it has been in contact with ammonia at a concentration of $16.7 \%$.

The threshold voltages, $V_{\mathrm{T}}$, and charge carrier mobilities, $\mu_{\text {sat }}$, were extracted in the saturation region of the characteristics. They are summarized in Table 1. In the pristine device, the interface is 'heavily' protonated by the sulfonic acid groups and hence a large positive voltage of more than $70 \mathrm{~V}$ is necessary to deplete the channel. Upon the controlled exposure to ammonia, the sulfonic acid groups at the interface are transformed to ammonium sulfonates according to Fig. 1. In line with the applied ammonia dose, the threshold voltage decreases in steps of about $10 \mathrm{~V}$ over a wide range of more than $90 \mathrm{~V}$. The mean value of the extracted mobilities is $1.2 \times 10^{-4} \mathrm{~cm}^{2} / \mathrm{V} \mathrm{s}$ with a standard deviation of less than $10 \%$, which means that the mobility is virtually not affected by the gas exposure. Also, when repeating the experiment, variations of the obtained threshold voltages upon exposure to the same ammonia doses were only a few volts as shown in Fig. 5, which summarizes the obtained threshold voltages for two sets of experiments in a bar diagram.

As can be shown by drift-diffusion-based device modelling [19, 20], the threshold voltage shift can be associated with a density of fixed charges at the interface (in our case the $-\mathrm{SO}_{3}^{-}$groups) via

$\Delta V_{\mathrm{th}}=-\frac{\sigma_{\mathrm{if}}}{C_{\mathrm{ox}}}$.

Here, $\Delta V_{\text {th }}$ is the threshold voltage shift, $\sigma_{\text {if }}$ the interface charge density, and $C_{\mathrm{ox}}$ the capacitance per unit area of the gate oxide. For the devices used, the specific capacity of the gate oxide can be estimated to be $209 \mu \mathrm{F} / \mathrm{m}^{2}$. Assuming that the threshold voltage without any charge carriers present at the interface is $0 \mathrm{~V}$, the density of fixed interface charges contained in Table 1 can be extracted. The exact origin of the negative threshold voltages after extended exposure to $\mathrm{NH}_{3}$ is not fully understood. Considering, however, that also in devices without any interfacial layer, slightly more negative threshold voltages are observed after exposure to $\mathrm{NH}_{3}$ [14], they are attributed to (irreversible) interactions between $\mathrm{NH}_{3}$ and either the active material or the gold electrodes.

To test the long-term stability of the threshold voltage shift induced by the $\mathrm{NH}_{3}$ exposure, the devices after the last exposure step were stored in argon atmosphere and measured again after several days. As can be seen in Fig. 6, after an initial relaxation of the device (observed when comparing the original characteristics with those after 5 days), further changes after 7 and 11 days were only very minor. 

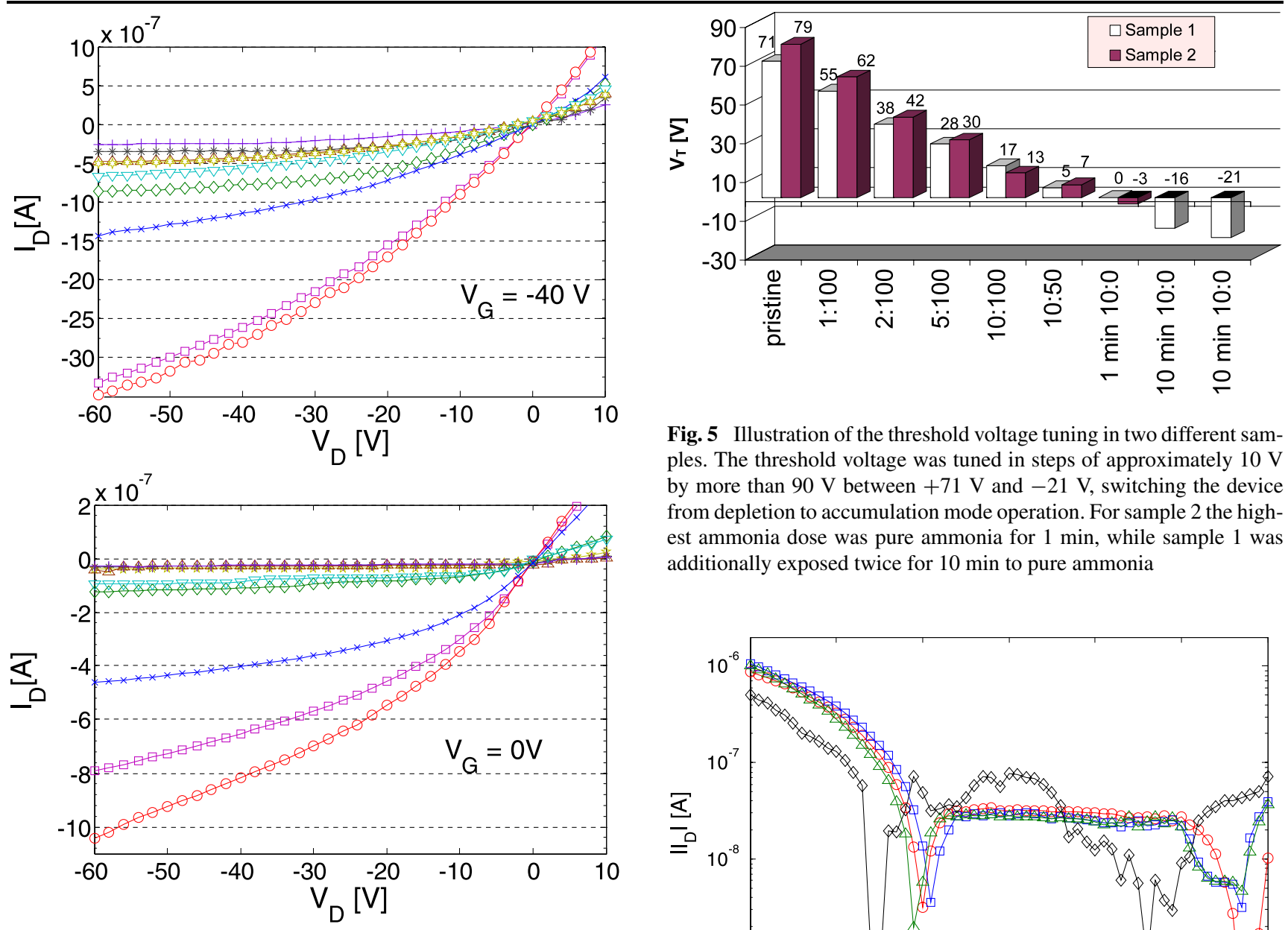

Fig. 5 Illustration of the threshold voltage tuning in two different samples. The threshold voltage was tuned in steps of approximately $10 \mathrm{~V}$ by more than $90 \mathrm{~V}$ between $+71 \mathrm{~V}$ and $-21 \mathrm{~V}$, switching the device from depletion to accumulation mode operation. For sample 2 the highest ammonia dose was pure ammonia for $1 \mathrm{~min}$, while sample 1 was additionally exposed twice for $10 \mathrm{~min}$ to pure ammonia

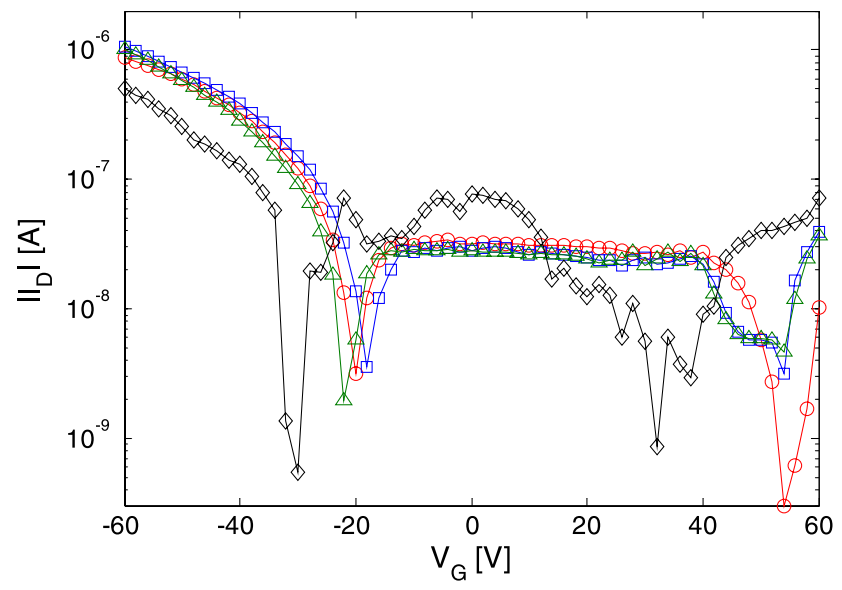

Fig. 4 Output characteristics of a rr-P3HT OTFT containing a reactive T-SC/SA layer at gate voltages of - $40 \mathrm{~V}$ (upper) and $0 \mathrm{~V}$ (lower), after successive exposure to ammonia at different concentrations. At a gate voltage of $-40 \mathrm{~V}$ the device is switched on for all cases, while at $0 \mathrm{~V}$ gate voltage the device is switched off after it has been in contact with ammonia at a concentration of 10:50 (16.7 vol.\%) (red circles: prior to exposure to $\mathrm{NH}_{3}$, purple rectangles: after exposure to ammonia to argon ratio of 1:100 (a flow of $1 \mathrm{~L} / \mathrm{h} \mathrm{NH}_{3}$ and $100 \mathrm{~L} / \mathrm{h} \mathrm{Ar}$ ) for $1 \mathrm{~min}$, blue x's: after 1 min of a 2:100 mixture, green diamonds: after $1 \mathrm{~min}$ of a 5:100 mixture, turquoise triangles: after $1 \mathrm{~min}$ of a 10:100 mixture, yellow stars: after $1 \mathrm{~min}$ of a 10:50 mixture, brown triangles: after $1 \mathrm{~min}$ of a 10:0 mixture, black crosses: after $10 \mathrm{~min}$ of a 10:0 mixture, violet pluses: after another $10 \mathrm{~min}$ of a 10:0 mixture)

\section{Summary and conclusions}

The results presented in this contribution show that a chemically reactive layer at the semiconductor-dielectric interface allows the realization of an OTFT whose threshold voltage can be tuned after assembly over a wide range. This is achieved by a strong local doping of the interfacial region of the organic semiconductor by sulfonic acid groups attached to a siloxane layer bonded to the silicon oxide gate dielectric. This doping can be gradually compensated by exposure to ammonia, which leads to the formation of the corresponding ammonium sulfonate at the interface. Carefully choos-

Fig. 6 Transfer characteristics of a rr-P3HT OTFT containing a reactive T-SC/SA layer directly after 'full' $\mathrm{NH}_{3}$ exposure (black diamonds) as well as measured after 5 (red circles), 7 (blue squares), and 11 (green triangles) days

ing the ammonia dose then allows a controlled change of the threshold voltage of a poly(thiophene)-based transistor after exposure in steps of, for example, approx. $10 \mathrm{~V}$ from $>+70 \mathrm{~V}$ to $<-20 \mathrm{~V}$. This procedure does not lead to any degradation of the device characteristics and the threshold voltage shift achieved after the highest $\mathrm{NH}_{3}$ dose is stable over many days.

Acknowledgements Financial support by the Austrian Science Fund FWF through Project No. P19959-N20 and in the framework of the Austrian Nano-Initiative (Research Project Cluster 0700Integrated Organic Sensor and Optoelectronics Technologies-Research Project No. 0701) is gratefully acknowledged. The authors are grateful to S. Possanner and K. Zojer for stimulating discussions and to Plextronics Inc. for free samples of rr-P3HT (Plexcore OS1100) used in initial experiments. 
Open Access This article is distributed under the terms of the Creative Commons Attribution Noncommercial License which permits any noncommercial use, distribution, and reproduction in any medium, provided the original author(s) and source are credited.

\section{References}

1. C. Dimitrakopoulus, D. Mascaro, IBM J. Res. Dev. 45, 1 (2001)

2. F. Assaderaghi, D. Sinitsky, S. Parke, J. Bokor, P. Ko, C. Hu, in IEEE International Electron Devices Meeting (IDEM), 1994, pp. $809-812$

3. H. Kotaki, S. Kakimoto, M. Nakano, T. Matsuoka, K. Adachi, K. Sugimoto, T. Fukushima, Y. Sato, in IEEE International Electron Devices Meeting (IDEM), 1996, pp. 459-462

4. M. Miyazaki, G. Ono, T. Kawahara, in Circuits and Systems (ISCAS 2005), vol. 1, pp. 17-20

5. J. Kedzierski, D. Boyd, P. Ronsheim, S. Zafar, J. Newbury, J. Ott, C. Cabral, M. Ieong, W. Haensch, in Electron Devices Meet., 2003 (IEEE International, New York, 2003), pp. 13.3.1-13.3.4

6. S. Iba, T. Sekitani, Y. Kato, T. Someya, Appl. Phys. Lett. 87, 023509 (2005)

7. M. Scharnberg, V. Zaporojtchenko, R. Adelung, F. Faupel, Appl. Phys. Lett. 90, 013501 (2007)

8. G. Maltezos, R. Nortrup, Appl. Phys. Lett. 83, 102067 (2003)

9. A. Wang, I. Kymissis, V. Bulović, A. Akinwande, IEEE Trans. Electron Devices 53, 1 (2006)
10. A. Wang, I. Kymissis, V. Bulović, A. Akinwande, Appl. Phys. Lett. 89, 112109 (2006)

11. Y. Guo, Y. Liu, C. Di, G. Yu, W. Wu, S. Ye, Y. Wang, X. Xu, Y. Sun, Appl. Phys. Lett. 91, 263502 (2007)

12. K.P. Pernstich, S. Haas, D. Oberhoff, C. Goldmann, D.J. Gundlach, B. Batlogg, A.N. Rashid, G.J. Schitter, J. Appl. Phys. 96, 6431 (2004)

13. S. Kobayashi, T. Nishikawa, T. Takenobu, S. Mori, T. Shimoda, T. Mitani, H. Shimotani, N. Yoshimoto, S. Oagawa, Y. Iwasa, Nature Mater. 3, 317 (2004)

14. P. Pacher, A. Lex, V. Proschek, H. Etschmaier, E. Tchernychova, M. Sezen, U. Scherf, W. Grogger, G. Trimmel, C. Slugovc, E. Zojer, Adv. Mater. 20, 3143 (2008)

15. C. Goldmann, C. Krellner, K.P. Pernstich, S. Haas, D.J. Gundlach, B. Batlogg, J. Appl. Phys. 99, 034507 (2006)

16. C. Tanase, E.J. Meijer, P.W.M. Blom, D.M. de Leeuw, Org. Electron. 4, 33 (2003)

17. M. Mottaghi, G. Horowitz, Org. Electron. 7, 528 (2006)

18. P. Pacher, A. Lex, V. Proschek, O. Werzer, P. Frank, S. Temmel, W. Kern, R. Resel, A. Winkler, C. Slugovc, R. Schennach, G. Trimmel, E. Zojer, J. Phys. Chem. C 111(33), 12407 (2007)

19. S. Scheinert, K.P. Pernstich, B. Batlogg, G. Paasch, J. Appl. Phys. 102, 104503 (2007)

20. S. Possaner, K. Zojer, P. Pacher, E. Zojer, F. Schürrer, Adv. Funct. Mater. (2008). doi:10.1002/adfm.200801466 